\title{
Development of superovulation program and heterologous in vitro fertilization test assessment in hamsters.
}

\begin{abstract}
Superovulation has become a common assisted reproductive technology in the field of animal reproduction. In addition, zona-free hamster oocytes have been used in heterologous in vitro fertilization research to evaluate sperm function. A study was conducted to compare eight different superovulation protocols for golden hamsters using two concentrations of human Chorionic Gonadotropin (hCG) given at two time intervals post-pregnant mare's serum gonadotrophin (PMSG) injection and two time intervals of oocyte harvesting. Fifty-six female golden hamsters were randomly and equally assigned into eight superovulation groups. Hamsters were superovulated initially with PMSG followed by human Chorionic Gonadotrophin (hCG). All the groups received 40 IU PMSG, either 40 or $45 \mathrm{IU}$ hCG given at either 48-50 or 55-57 h post PMSG injection and the oocytes recovered at either 12-15 or 16$18 \mathrm{~h}$ after hCG injection. Higher number of recovered oocytes $(51.57 \pm 0.83)$ and maturation rates $(94.20 \%)(\mathrm{p}<0.05)$ were detected in hamsters which received $45 \mathrm{IU}$ hCG at $55-57 \mathrm{~h}$ after PMSG injection when the oocytes were recovered later at 16-18 $\mathrm{h}$ compared with hamsters in the other groups. Mean fertilization rate of hamsters given $45 \mathrm{IU}$ hCG at 55-57 h post PMSG injection ranged from $77.89-78.84 \%$ and were significantly higher $(\mathrm{p}<0.05)$ than those that received hCG at 48-50 h post PMSG injection. In conclusion administration of 40 IU PMSG followed by $45 \mathrm{IU}$ hCG injection at 55 and $57 \mathrm{~h}$ post PMSG injection followed by oocyte recovery after 16-18 $\mathrm{h}$ gave the highest response in oocyte recovery and maturation in golden hamsters.
\end{abstract}

Keyword: Hamster; HCG; PMSG; Superovulation; Zona-free hamster oocytes 